\title{
Alexander Burnes, un héritier des Lumières écossaises dans le sous-continent indien à l'âge des réformes
}

Alexander Burnes and the heritage of the Scottish Enlightenment in the Indian Subcontinent in the Age of Reform

\section{Nadine André}

\section{OpenEdition}

Journals

Édition électronique

URL : https://journals.openedition.org/etudesecossaises/254

DOI : 10.4000/etudesecossaises.254

ISSN : 1969-6337

Éditeur

UGA Éditions/Université Grenoble Alpes

Édition imprimée

Date de publication : 31 mars 2011

Pagination : 33-48

ISBN : $978-2-84310-191-5$

ISSN : $1240-1439$

\section{Référence électronique}

Nadine André, «Alexander Burnes, un héritier des Lumières écossaises dans le sous-continent indien à l'âge des réformes », Études écossaises [En ligne], 14 | 2011, mis en ligne le 31 mars 2012, consulté le 11 avril 2023. URL : http://journals.openedition.org/etudesecossaises/254 ; DOI : https://doi.org/

10.4000/etudesecossaises.254 


\section{Alexander Burnes, un héritier des Lumières écossaises dans le sous-continent indien à l'âge des réformes}

Une tradition veut que l'Empire des Indes ait été conquis par les soldats irlandais au bénéfice des marchands écossais ${ }^{1}$. Parlant de l'Empire britannique, l'Écossais James Mill a déclaré qu'il constituait «a vast system of outdoor relief for the upper classes». C'est particulièrement le cas pour les Écossais et les protestants nord-irlandais. Le rôle joué par les Écossais dans l'Empire à la suite de l'adoption de l'Acte d'Union a été évoqué par Linda Colley dans Britons: Forging the Nation, 1707-1837. Dans British India and British Scotland, 1780-1830, Martha McLaren a évalué l'héritage des Lumières écossaises chez Thomas Munro, Mountstuart Elphinstone et John Malcolm².

Nombreux sont les serviteurs de l'East India Company dont l'héritage écossais mérite d'être évalué. Ainsi Alexander Burnes qu'une génération sépare de ses illustres prédécesseurs. À la phase de construction de l'Empire britannique dans le sous-continent a succédé celle de la consolidation. Il y a plus que jamais des emplois à pourvoir dans l'armée et l'administration. Mais les dangers qui menacent le sous-continent n'ont pas disparu, bien au contraire. On redoute l'ennemi intérieur tout autant que celui qui guette à la périphérie, qu'il soit asiatique ou européen. C'est sur le terrain de la politique diplomatique et territoriale que Burnes va s'illustrer.

La carrière d'Alexander Burnes n'a, à notre connaissance, jamais été présentée en partant de ses origines et de sa culture écossaises. On tentera donc d'évaluer en quoi son parcours professionnel se distingue de celui des Écossais de la génération précédente et à quels problèmes il est confronté, avant de considérer ses vues en matière de politique

1. F. Tennyson Jesse, Story of Burma, Londres, Macmillan, 1946, p. 4.

2. Thomas Munro est gouverneur de Madras de 1819 à 1827, Mountstuart Elphinstone gouverneur de Bombay de 1819 à 1827 et John Malcolm gouverneur de Bombay de 1827 à 1830 . 
territoriale. On verra enfin dans quelle mesure il est imprégné des valeurs des Lumières écossaises.

\section{Origines et parcours professionnel}

Comme pour ses prédécesseurs, l'Inde offre à Alexander Burnes (18051841), issu de la classe moyenne, les opportunités que ne lui offrent ni l'Écosse, ni l'Angleterre. Apparenté au poète Robert Burns, chantre du nationalisme écossais, Alexander naît à Montrose dans une famille de la petite bourgeoisie. Son père a occupé les postes de secrétaire de mairie, puis de maire de la ville. Burnes mène à la Trades School une scolarité correcte, se distinguant davantage par son énergie que par ses talents scolaires. Il a conscience d'avoir été un élève médiocre : «I am the only illiterate man in my family_all professions but me $^{3}$.» Avec cinq fils et quatre filles, son père fait tout naturellement appel à Joseph Hume, lui aussi originaire de Montrose, pour qu'il fasse entrer ses fils au service de la Compagnie ${ }^{4}$. Alexander est nommé élève officier dans l'armée de la présidence de Bombay où il arrive en 1821. Il a l'âge (seize ans) et le parcours classique des nouveaux arrivants. Il est accompagné de son frère James, qui fera carrière comme médecin dans la présidence de Bombay et publiera le récit de sa visite aux émirs du Sind en 1828.

Burnes a bénéficié pour sa nomination des anciennes techniques d'avancement décrites par M. McLaren que sont les relations et le «patronage» (clientélisme ou parrainage) mais, une fois en Inde, il est confronté à la rigidité du système d'avancement à l'ancienneté en vigueur dans l'armée de la Compagnie. Le seul moyen de contourner ce système consiste à avoir des relations très bien placées ou à se distinguer pour être détaché de l'armée et affecté soit au service politique, soit dans l'administration. Le recours au détachement est rendu nécessaire par le nombre très limité de fonctionnaires appartenant au prestigieux Indian Covenanted Civil Service (800 en moyenne).

S'il veut que sa carrière lui apporte honneurs et reconnaissance financière, Burnes dépend donc de ses seules compétences. Or il débute sa carrière au moment où, dans le sous-continent, les conflits offrant des

3. J. W. Kaye, Lives of Indian Officers, vol. 2, p. 11.

4. De 1897 à 1808, Joseph Hume a fait une brillante carrière au sein de la Compagnie. Fortune faite, il est rentré en Grande-Bretagne et a entamé en 1812 une carrière politique qui l'a vu se rapprocher de Jeremy Bentham et de James Mill. Élu à la Chambre des communes, il s'est distingué par des convictions de radical, de réformateur et de philanthrope. Il est le père d'Allan Octavian Hume, le fonctionnaire de l'Indian Civil Service qui participe à la fondation de l'Indian National Congress en 1885. 
perspectives de promotion rapide aux jeunes officiers sont devenus rares. Le Directoire comme le gouvernement ${ }^{5}$ s'opposent de longue date à toute nouvelle expansion territoriale. Burnes a sous les yeux l'exemple d'Elphinstone, alors gouverneur de Bombay, et de John Malcolm. En l'absence de relations, ou lorsque celles-ci ne suffisaient plus, c'est en publiant ouvrages et rapports officiels sur les affaires de l'Inde ou des pays voisins qu'ils sont finalement parvenus à accéder aux plus hautes fonctions.

Burnes est tout d'abord affecté au $1^{\mathrm{er}}$ bataillon du $3^{\mathrm{e}}$ régiment d'infanterie indigène de Bombay. L'armée de la Compagnie est moins prestigieuse que celle de la reine mais, comme le remarque J. Lunt, l'armée de Bombay est plus petite que celle des deux autres présidences et une jeune recrue a plus de chances de s'y distinguer. Pour obtenir de l'avancement et son détachement de l'armée, Burnes s'emploie donc à mettre en œuvre les nouvelles techniques d'avancement de carrière : maîtrise des langues orientales, expertise, production de rapports et autopromotion. Elles s'appliquent d'ailleurs en Grande-Bretagne aussi où John Mill doit à son Histoire de l'Inde (1818) d'être recruté par la Compagnie en 1823. Burnes, qui n'aime pas l'exercice physique mais est attiré par l'écriture («I ought not to have been a soldier, although I glory in the profession, for I am too fond of pen and $\left.i n k^{6} »\right)$, manifeste dès son arrivée des dispositions particulières pour les langues orientales, apprenant l'hindoustani, puis le persan, ce qui lui vaut des promotions rapides à des postes d'interprète. En avril 1825, des troupes venues du Sind voisin mènent une incursion au Cutch, alors État princier protégé, où il est affecté. Le corps auquel il est rattaché ne prenant pas part à la campagne, Burnes se consacre à l'exploration, ce qui lui vaut d'être nommé à l'état-major général du capitaine Pottinger, le résident. C'est désormais avec la rédaction de rapports, la collecte de statistiques et un travail topographique et cartographique qu'il se distingue. Les rapports et mémoires qu'il rédige lui valent des récompenses financières ainsi que l'estime de ses supérieurs ${ }^{7}$. Il est une nouvelle fois promu en 1828 et affecté au quartier général de l'armée à Bombay où il rencontre le gouverneur de la présidence, John Malcolm.

5. Hérité de la tradition whig, le «double government» repose sur un système de poids et de contrepoids, le Directoire et l'India Board (ou Board of Control) étant censés s'équilibrer mutuellement. Mais dans la pratique, le Directoire gère les affaires courantes tandis que toutes les décisions d'importance sont prises par le Cabinet où le ministre des Affaires étrangères prime sur le président de l'India Board.

6. J. W. Kaye, Lives of Indian Officers, vol. 2, p. 12.

7. «The statistics of Wagur» (janvier 1827), ainsi qu'un rapport sur l'embouchure orientale du delta de l'Indus (1828). 
Le renseignement militaire est une activité dangereuse, mais qui permet une évolution de carrière rapide. Burnes est ambitieux et courageux. N'a-t-il pas une fascination quasi enfantine pour Alexandre le Grand? Ses relations de voyage comportent de fréquentes références au conquérant mythique et il n'a de cesse de découvrir dans les territoires qu'il traverse des traces de son passage. Il se porte donc volontaire pour assurer des missions d'exploration dans des régions dont il sait qu'elles attisent la curiosité des autorités. Dans un premier temps, il bénéficie autant qu'il est possible de la solidarité entre Écossais. Avec le soutien du résident du Cutch et de John Malcolm, Burnes demande en 1829 l'autorisation d'explorer le Rajputana. Le gouverneur général, Lord Bentinck (1828-1835), donne son accord avant de changer d'avis. À peine parti, Burnes doit rebrousser chemin. Charles Metcalfe, alors membre du Conseil suprême du gouvernement de l'Inde (1827-1835), a, selon Lunt, fait valoir qu'il était inutile d'attiser la suspicion des émirs du Sind ainsi que celle des princes rajputs. Homme de la génération de Malcolm et d'Elphinstone, Metcalfe est une autorité morale en Inde. Cela n'empêche pas la carrière de Burnes de continuer à évoluer favorablement. En septembre 1829, il obtient son affectation, tant attendue, au service politique et est nommé assistant du résident. Il participe également à une étude de la frontière du nord-ouest et est invité par le Directoire à achever une carte du Cutch entamée peu avant.

En 1831, Malcolm lui confie la direction d'une mission diplomatique auprès de Ranjit Singh, à Lahore. Il est prévu que le voyage s'effectue par voie fluviale, sur l'Indus, alors que les émirs du Sind sont hostiles à toute présence étrangère sur leur territoire. Opposé au projet dont le motif officiel n'abuse personne, Charles Metcalfe n'a pas, cette fois-ci, gain de cause. La mission est l'occasion de rédiger un rapport très complet sur les possibilités de navigation sur le fleuve qui donne accès, au-delà du Sind et du Béloutchistan, au Punjab, au Cachemire, à l'Afghanistan et à l'Asie centrale, sur la situation politique, les effectifs militaires, les perspectives commerciales, les ressources naturelles et quantité d'autres sujets.

Reçu à Simla par Bentinck, Burnes est félicité pour son rapport. C'est l'occasion rêvée de présenter le projet qui lui tient à cœur, un périple qui le verrait traverser le royaume de Kaboul, les khanats d'Asie centrale, Boukhara notamment, jusqu'à la Caspienne. Le gouvernement britannique s'inquiète de la présence russe dans la région qui est mal connue et inhospitalière. Son projet ne pouvait mieux tomber. Le périple est la mission d'exploration par excellence. En dehors du royaume de Kaboul et de la Perse, Burnes voyage sous différentes identités. Après avoir pris la route le 3 janvier 1832, il rallie Bombay le 18 janvier 1833 via Bushire 
après avoir atteint la Caspienne et traversé la Perse où il a rencontré le Shah ainsi que le prince héritier.

Porteur de ses propres dépêches destinées à l'India Board, Burnes arrive à Londres au début du mois d'octobre 1833. Sollicité de toutes parts, celui que l'on surnomme désormais «Bokhara Burnes» est reçu par le président du Board of Control, Charles Grant, le Premier ministre, Lord Grey, et Guillaume IV. Il tire du rapport officiel de sa mission des articles ainsi qu'un récit que l'autorise à publier le Board of Control. Paru en 1834, l'ouvrage est un succès de librairie immédiat. Ses efforts sont récompensés par son élection en qualité de membre par la Royal Asiatic Society. La Royal Geographical Society lui décerne la médaille d'or. Ses exploits sont célébrés à Paris également où, en décembre 1834, Burnes est invité par la Société géographique qui lui décerne la médaille d'argent. Il donne de nombreuses conférences, notamment devant des sociétés savantes. De passage à Montrose, l'enfant du pays est reçu avec les honneurs. Aux deux établissements scolaires de la ville, il fait une donation qui contribuera au financement des prix destinés aux meilleurs étudiants.

Soucieux de se ménager les faveurs des plus hautes autorités du gouvernement de l'Inde, Burnes leur dédicace les Voyages à Boukhara. Le volume 1 est dédié à Bentinck et le volume 3 à la mémoire de Malcolm. Il veille aussi dans ce volume à exprimer sa gratitude à Elphinstone qui l'a fait bénéficier de ses conseils alors qu'il préparait la publication de son récit, citant à plusieurs reprises l'ouvrage publié par ce dernier sur l'Afghanistan $^{8}$. Enfin, dans l'introduction du volume 3, Burnes mentionne le commandant en chef de l'armée de Bombay qui lui a signalé l'intérêt que pouvait présenter la frontière du nord-ouest. En 1841, lorsqu'il prépare la publication du récit de sa mission à Kaboul en 18361838, mission qui a échoué, Burnes n'a plus de puissant protecteur à remercier. Aussi Kaboul, préparé en 1841 et publié de manière posthume en 1842, est-il dédié à son père.

Burnes fait des choix qui révèlent ses priorités. En 1832, il a décliné la proposition faite par Dost Mohammad, souverain de Kaboul, de commander son armée. En 1834, jeune officier célibataire promis à une brillante carrière et reçu dans la meilleure société, Burnes qui, il est vrai, est connu pour son goût pour la gent féminine, ne se marie pas. À la suite des multiples contacts qu'il a lors de son séjour en Grande-Bretagne, des propositions de postes lui sont faites, dans la diplomatie notamment.

8. M. Elphinstone, An Account of the Kingdom of Caubul and its Dependencies in Persia, Tartary and India, Comprising a View of the Afghaun Nation and a History of the Dooraunee Monarchy, 1815. 
Il choisit néanmoins de repartir pour le sous-continent où des promotions régulières couronnent ses efforts. Il a entre temps obtenu la nomination de son frère cadet, Charles, dans l'armée de Bombay.

Les talents dont Burnes a fait preuve dès le début de sa carrière lui ont valu de bénéficier de la bienveillance de ses supérieurs écossais et de leurs réseaux. Mais lorsqu'il reprend son poste d'assistant du résident du Gutch en 1835, la situation a changé : Malcolm est décédé et Elphinstone est rentré en Grande-Bretagne. Le nouveau gouverneur général, Lord Auckland (1836-1842), est influencé à la fois par Palmerston, alors ministre des Affaires étrangères, et par William Macnaghten, secrétaire du Conseil suprême qui a fait toute sa carrière au service de la Compagnie à Calcutta. Faute d'avoir aidé la Perse à se prémunir contre la Russie par le biais de la diplomatie européenne, les autorités britanniques décident de faire endosser à l'Afghanistan le rôle d'État tampon contre toute avancée russe en direction du sous-continent. La mission qu'accomplit Burnes à Kaboul a des objectifs à la fois politiques, diplomatiques, commerciaux et géographiques. Mais elle est vouée à l'échec, moins du fait de la présence simultanée dans la capitale d'une mission russe que du refus d'Auckland de faire la moindre concession. Burnes se retrouve en désaccord avec ses supérieurs sur l'appréciation de la situation, le rôle que doit jouer l'Afghanistan (il défend un État unifié fort avec à sa tête Dost Mohammad tandis qu'Auckland opte pour un État divisé et faible dirigé par un souverain fantoche) et le choix de l'homme qui sera leur allié le plus fiable (Dost Mohammad ou Shah Shudja). Paradoxalement, la politique menée par Palmerston en 1838 comme en 1855 (il est alors Premier ministre) tend à faciliter le travail de sape mené par la Perse et la Russie puisqu'il est beaucoup plus simple de s'implanter dans un Afghanistan divisé.

C'est alors que Burnes fait des choix qui ont donné matière à discussion. Après avoir un temps envisagé de démissionner s’il n’était pas nommé Envoyé du gouverneur général auprès de Shah Shudja à Kaboul (le poste va à Macnaghten), et sans doute parce qu'il ne veut pas être mis à l'écart, Burnes accepte d'être l'assistant de celui-ci. Il est vrai que la campagne est une occasion inespérée pour beaucoup de militaires, mais aussi de fonctionnaires civils, d'obtenir de l'avancement et des honneurs. Ses supérieurs lui font miroiter des responsabilités à la hauteur de ses espérances une fois la position de Shah Shudja affermie. En attendant, il est promu au grade de lieutenant-colonel honoraire et est anobli. Il prend une part active à la campagne afghane et semble soudain acquis aux vues de Macnaghten. Mais ses avertissements, ainsi que ceux d'autres officiers expérimentés, sur l'essentiel, à savoir que Shah Shudja n'a pas le soutien de ses sujets et que la position britannique à Kaboul est périlleuse, sont 
délibérément ignorés. Les erreurs politiques et stratégiques se succèdent. En septembre 1841, Macnaghten apprend sa nomination au poste de gouverneur de Bombay. Il laisse entendre à Burnes qu'il lui succédera au poste d'Envoyé. Tous deux ont intérêt à maintenir jusqu'au départ de Macnaghten la version officielle selon laquelle le calme règne en Afghanistan. L'insurrection débute le 2 novembre 1841 avec le meurtre de Burnes et de son frère, le lieutenant Charles Burnes. Alexander n'a, il faut bien le dire, guère pris de précautions malgré plusieurs avertissements. Sans doute savait-il déjà que tout était perdu. Apparemment fataliste, il a refusé de se réfugier dans le cantonnement au prétexte que cela donnerait l'impression qu'il avait peur et pensait qu'il était temps que les Britanniques quittent le pays. La retraite de Kaboul est un désastre dont la portée résonne bien au-delà des frontières de l'Afghanistan. La crainte des attentistes, dont Charles Metcalfe, qui estimaient que toute implication britannique accrue en Afghanistan, même pour des raisons commerciales, les entraînerait inévitablement dans une situation politique dont la portée leur échapperait, se réalise. Pour la première fois de son histoire dans le sous-continent, l'armée de la Compagnie est anéantie, et son prestige avec. C'est la légitimité même du Raj qui est remise en cause.

\section{Position en matière de politique territoriale}

Les années 1830 sont une période où l'on prône à la fois les réformes et la maîtrise des dépenses («retrenchment»). Le programme de réformes préconisé par l'India Act de 1833 influencé par James Mill est appliqué a minima pour des raisons d'économies. La gestion des questions politiques et territoriales pâtit également de ces restrictions budgétaires.

À l'intérieur du sous-continent, tous les États princiers sont désormais liés par traité à la Compagnie et sont soit des états subsidiaires, soit des protectorats. Seule demeure la crainte d'un ennemi intérieur et/ou de troubles fomentés par des puissances étrangères. Paternalistes et utilitaristes s'affrontent quant au rôle que doivent jouer ces États. E. Stokes évoque l'hostilité non déguisée de James Mill vis-à-vis des princes dont il considère le gouvernement comme oppressif, voire corrompu ${ }^{9}$. L'intérêt général doit prévaloir. Les annexions bénéficieront à l'ensemble de la population. Toute occasion d'abolir ces États doit donc être mise à profit et le plus tôt sera le mieux. Quelques années plus tard, K. Marx

9. E. Stokes, The English Utilitarians and India, p. 250. 
fait la même analyse ${ }^{10}$. De leur côté, les paternalistes s'accordent globalement pour considérer que le gouvernement britannique est meilleur que le gouvernement indigène, mais refusent le recours à l'annexion. La politique des traités en vigueur depuis le début du siècle repose sur une administration indirecte et récuse, en théorie, toute ingérence dans les affaires internes des États princiers. Mais, sous l'influence des paternalistes représentés par Malcolm, l’ingérence est en réalité de plus en plus forte. On évite ainsi en apparence toute nouvelle expansion territoriale tout en s'assurant la soumission des États concernés. Dans un premier temps, Burnes ne remet pas en cause la politique des traités et se laisse volontiers tenter par l'ingérence, comme le montre son projet de voyage au Rajputana. Cette volonté de ne pas abolir les gouvernements indigènes s'explique en partie par la tradition écossaise d'ouverture vis-à-vis des cultures étrangères, orientale ou asiatique en l'occurrence. L'adoption de l'India Act de 1833 lui porte un coup fatal. Pour leur part, les représentants d'un autre courant du paternalisme incarné par Metcalfe évoluent pour devenir moins réticents à mettre un terme au gouvernement indigène. Depuis 1830 est également mise en œuvre une politique officielle d'absorption territoriale par des moyens pacifiques en vertu du principe de souveraineté («paramountcy») de la Compagnie. Lord Dalhousie, gouverneur général de 1848 à 1856, théorisera cette politique dont il n'est nullement l'initiateur sous le nom de doctrine de la déshérence («lapse»), ce qui amène certains à lui en attribuer à tort la paternité.

Autre aspect de cette politique intérieure, la manière dont sont considérés les anciens souverains, symboles auxquels les Indiens restent très attachés. Burnes évolue à une époque charnière. Il semble avoir, dans le cas de Shah Shudja, roi déposé d'Afghanistan (1803-1809) et pensionnaire de la Compagnie réfugié en territoire britannique, et avant la première guerre afghane, partagé une vision romantique burkéenne des souverains déchus encore assez courante à l'époque chez les paternalistes. Préserver le décorum qui entoure les souverains, même lorsqu'ils ont perdu tout pouvoir, est considéré comme une manière de renforcer la légitimité du gouvernement de la Compagnie. Néanmoins, Burnes s'en distingue dans le même temps en qualifiant d'«absurde» le cérémonial qui entoure l'empereur moghol, pensionnaire de la Compagnie qui ne détient plus aucun pouvoir depuis longtemps, à qui il rend visite à Delhi en 1831 .

Vis-à-vis des États frontaliers indépendants, la politique se veut officiellement neutre. La menace française a été remplacée par la menace russe dont le gouvernement britannique bien plus que le gouvernement

10. K. Marx et F. Engels, «La question des Indes orientales», Du Colonialisme en Asie, p. 40-41. 
de l'Inde, qui doit faire face à des dangers plus immédiats, commence à s'inquiéter au début des années 1830.

Dès sa première mission, qui l'amène à traverser le Sind, Burnes ne défend pas une politique expansionniste au sens strict mais fait siennes les recommandations de William Moorcroft (1770 [?]-1825), vétérinaire nommé à la tête des haras de la Compagnie et explorateur des pays limitrophes de la frontière du nord-ouest, en matière de développement commercial pour concurrencer les marchands russes. Le projet d'établissement d'une foire sur les rives de l'Indus avait également retenu l'attention d'Ellenborough lorsqu'il occupait le poste de président de l'India Board dans le gouvernement Wellington (1828-1830). Burnes souscrit au libéralisme et encourage une politique interventionniste en insistant sur les perspectives de développement commercial dans la région. Le commerce n'est-il pas considéré par David Hume et Adam Smith comme un facteur de progrès, notamment en termes de droits de l'individu, et un moyen d'exercer une influence plus efficace que la conquête? Peu importe que, dans l'Empire, le commerce soit source de disputes qui, parfois, dégénèrent en conflit armé. Les relations entre les marchands britanniques et la Chine à la même époque constituent pourtant un cas d'école édifiant. Burnes, qui a une carrière à construire, a en commun avec ses prédécesseurs de correspondre au portrait que brosse L. Colley qui rappelle la propension à l'agressivité des Écossais en quête à la fois d'un gain économique et d'une reconnaissance qui prendrait la forme de postes prestigieux ${ }^{11}$. Acteur majeur du Grand Jeu, Burnes contribue à l'expansion de l'Empire en même temps qu'il assure l'avancement de sa carrière.

En 1836, confronté à la question afghane, Auckland réaffirme à Dost Mohammad que le gouvernement de l'Inde ne souhaite pas s'immiscer dans les affaires des pays indépendants. En 1838, pourtant, il opte pour l'extension à un État frontalier de la politique des traités. Sa politique n'est pas seulement interventionniste mais aussi belliciste, puisqu'elle repose sur une invasion et une période d'occupation. Un choix d'autant plus surprenant que l'heure est plus que jamais aux économies. Il n'est bien sûr pas question d'annexer l'Afghanistan, mais de replacer sur le trône un souverain déposé mais légitime et de l'assister jusqu'à ce qu'il ait repris le contrôle de son royaume. Pourtant, sous couvert d'assistance, les agents politiques exercent une ingérence très négativement ressentie, jusqu'au soulèvement prévisible.

Pour sa part, Burnes recommande simplement d'accorder à Dost Mohammad l'aide du gouvernement de l'Inde. Il accepte néanmoins de

11. L. Colley, Britons, p. 132. 
participer à l'invasion et à l'occupation de l'Afghanistan, endossant le rôle d'un conseiller politique dont les recommandations sont délibérément ignorées par l'Envoyé. Malgré ses réserves, il se met au service, comme les Écossais de la génération précédente, d'une politique territoriale agressive. Pour autant, la période du «military fiscalism» est révolue : la campagne afghane est menée pour des raisons stratégiques et non plus pour financer d'autres conquêtes.

\section{Dans quelle mesure Alexander Burnes est-il inspiré par I'héritage des Lumières écossaises?}

Les membres de la Clapham Sect sont bien représentés au sein du Directoire, au gouvernement et au Parlement ${ }^{12}$. Charles Grant est l'un d'entre eux. Mais la Compagnie est tenue d'être neutre. Comme ses prédécesseurs, Burnes considère que les questions religieuses ne doivent pas interférer avec la gouvernance des territoires. Il ne partage pas le sens de la mission religieuse ou civilisatrice («muscular christianity») qui caractérise à l'époque nombre d'administrateurs ou d'officiers. Presbytérien, il a une position modérée et est en fait sujet au scepticisme. Il trouve, comme cela est courant à l'époque, l'islam moins répréhensible que l'hindouisme, associé aux superstitions. Il réprouve néanmoins certaines pratiques, notamment celle consistant à enlever des êtres humains pour les vendre en esclavage, qu'il qualifie de «contre-nature ${ }^{13}$.

C'est à son statut particulier que Burnes doit de n'être pas coupé de la population comme le sont de plus en plus les autres serviteurs de la Compagnie. Explorateur talentueux autant que soldat et diplomate, il a une réelle curiosité intellectuelle. Il n'aime rien tant que se mêler à la population locale sous toutes sortes de déguisements. Sur cette aptitude ainsi que sur son respect scrupuleux des coutumes des pays qu'il parcourt repose le succès de ses missions. Cela rend d'autant plus surprenant qu'il ait, pendant l'occupation de l'Afghanistan (1839-1841), multiplié conquêtes et festivités qui ne pouvaient que susciter le ressentiment de la population musulmane. Sa réputation de séducteur n’est plus à faire. Contraint à l'inactivité (il se décrit comme un «highly paid idler»), en excellente

12. Mouvement anglican apparu dans la dernière décennie du XVIII ${ }^{\mathrm{e}}$ siècle dont les fondateurs vivaient près de Clapham, dans le Surrey. Il doit son influence à l'appartenance de nombre de ses membres aux plus hautes sphères politiques. Le mouvement encourageait l'évangélisme et la réforme sociale. Il s'est fortement impliqué dans la lutte contre l'esclavage.

13. Le commerce des esclaves est aboli dans les colonies britanniques en 1807 et l'esclavage rendu illégal dans la plupart des territoires britanniques en 1833. Il est finalement aboli dans les territoires de la Compagnie en 1843. 
santé, il lit beaucoup et mène grand train. Plus généralement, la venue à Kaboul des familles des soldats et des officiers, la vie sociale, les fêtes où l'on consomme de l'alcool irritent les musulmans. Burnes a la sympathie habituelle des officiers politiques britanniques pour les races martiales, musulmanes en particulier. Il se sent à l'aise au milieu des Afghans dont on se plait souvent à rappeler que leurs traits physiques et de caractère se rapprochent de ceux des Européens. Nul doute que Burnes soit attaché à l'Afghanistan dont il goûte le climat et qui lui rappelle son Écosse natale : «[...] I cannot tell you how my heart leaps, to see all the trees and plants of my native land growing around me in this country ${ }^{14}$.»

Burnes ne mène pas une réflexion sur les principes philosophiques qui sous-tendent son action politique. Il n'est pas un idéologue. Il est fondamentalement pragmatique - à entendre ici non pas au sens d'absence de convictions mais «qui est susceptible d'applications pratiques», "qui accorde la première place à l'action, à la pratique, qui se fonde sur la réussite dans l'action». Il ne devient pas non plus l'administrateur qu'il aurait pu être en raison de l'interruption prématurée de sa carrière.

La charte de 1833 sonne le glas du despotisme oriental, source de tous les maux. Le gouvernement de l'Inde prend alors la forme d'un autoritarisme réformateur, ou despotisme éclairé, qui n'est plus inspiré, comme à la génération précédente, par un quelconque modèle «asiatique» filtré par la culture écossaise qu'évoque McLaren ${ }^{15}$. L'autoritarisme qui caractérise la gouvernance écossaise révélerait, selon L. Colley, un goût pour le pouvoir monarchique qui trouverait son origine dans des sympathies jacobites ${ }^{16}$. Mais c'est plutôt dans le programme paternaliste que trouve son origine l'autoritarisme auquel souscrit Burnes dans le domaine politique.

C'est dans les rapports officiels de Burnes et dans les récits qu'il en a tirés qu'apparaissent clairement les valeurs des Lumières écossaises dont il est imprégné. Ils soulignent la qualité de l'éducation reçue, et ce d'autant plus que Burnes a quitté l'école tôt et n'a pas eu l'occasion, comme Malcolm par exemple, de reprendre ses études par la suite, à l'occasion de congés, notamment. La lecture en est agréable. L'expression est simple et directe. Elle révèle son érudition et l'intérêt qu'il porte aux territoires qu'il traverse, à leurs habitants et à une multitude de sujets.

Ses missions d'exploration renvoient à la valeur accordée à l'expérience. Burnes a un intérêt marqué pour l'histoire et ses vestiges ainsi que pour la politique auxquelles il associe naturellement l'élément humain.

14. J. W. Kaye, Lives of Indian Officers, vol. 2, p. 23.

15. M. McLaren, British India and British Scotland, 1780-1830, p. 11.

16. L. Colley, Britons, p. 131-132. 
Il sait aussi se faire anthropologue pour décrire les mœurs asiatiques, parfois surprenantes pour un Occidental. Il porte rarement un jugement moral et, s'il laisse deviner ses convictions personnelles, il veille à rester en retrait.

Il met en œuvre une approche scientifique pour traiter de sujets pratiques: les missions sont l'occasion de recherches poussées dans le domaine de la géologie, la quête de ressources minérales, l'étude des ressources agricoles, de la navigabilité des fleuves, de l'irrigation, la géographie physique et humaine, la chimie, voire l'alchimie, qui passionne les Afghans. La plupart de ces sujets présentent un intérêt direct pour le développement du commerce. L'étude des voies de communication fluviales et terrestres, des places fortes, des effectifs militaires, de l'armement est, quant à elle, essentielle au renseignement militaire.

L'expertise est au cœur de sa démarche. Burnes s'entoure d'hommes expérimentés et fiables, ingénieurs, géomètres, médecins, dessinateurs. Il rend des services insignes dans le domaine du renseignement et de la cartographie. L'expertise prend aussi la forme d'une production littéraire avec ses contributions à des publications de sociétés savantes telles que le Fournal of the Asiatic Society of Bengal ou les Transactions of the Bombay Geographical Society.

La valeur accordée à l'approche scientifique par les philosophes des Lumières écossaises puis, à partir de 1830, par les tenants du Benthamisme, prend, en matière de gouvernance, la forme d'une collecte systématique de données et de la publication de statistiques sur tous les aspects de la société indienne. En effet, désormais coupés de la population et de leurs informateurs traditionnels, les Britanniques ne disposent plus ni de l'«affective knowledge» ni de la «patrimonial knowledge» définies par G. Bayly ${ }^{17}$. Ces données sont devenues indispensables à un État qui repose plus désormais sur une bureaucratie centralisée que sur l'armée. De nouveaux organismes ou services apparaissent. À Londres, la Royal Geographical Society est fondée en 1830, la Statistical Society en 1834. La Compagnie crée pour sa part en 1846 le Statistical Department. Les North Western Provinces constituent une province pionnière. Le territoire est quadrillé, des archives sont constituées où sont consignés tous les titres de propriété. La population et les naissances sont décomptées. Le système a ses limites : la population n'apprécie pas nécessairement cette intrusion dans sa vie quotidienne. Bayly évoque aussi la «dual economy of knowledge» : si les Britanniques ont une connaissance de l'état de la société et des contacts relativement satisfaisants en zone urbaine, c'est loin d'être le cas dans les zones rurales éloignées qui constituent la majeure partie

17. C. Bayly, Empire and Information, p. 7-8. 
du territoire et auprès des classes les moins élevées de la société avec qui ils n'ont pratiquement pas de $\operatorname{contacts}^{18}$. La question se pose de savoir si cette collecte de statistiques est un facteur de progrès et de liberté ou d'asservissement. En fait, la pression exercée par la bureaucratie britannique n’a pas que des côtés négatifs. Cette collecte a, selon Bentham, le mérite de contrebalancer le risque de concentration du pouvoir entre les mains d'un seul individu qu'implique la centralisation ${ }^{19}$. Par ailleurs, elle permet aussi de vérifier que le sati et l'infanticide des filles sont des pratiques enrayées. Quant à l'archivage des titres de propriété, c'est l'assurance que les droits des plus humbles seront protégés. B. B. Misra y voit un apport capital en matière de liberté individuelle ${ }^{20}$.

Même si Burnes conserve un attachement très fort pour ses origines et sa culture écossaises, comme le révèle sa correspondance privée, son identité de citoyen britannique et sa loyauté envers la Grande-Bretagne ne font aucun doute. Il ne remet pas en cause la supériorité des Britanniques sur les peuples qu'ils gouvernent. Pourtant, il mesure vite les limites de sa notoriété acquise au service de l'Empire lorsqu'il est mis en demeure de servir une politique vouée à l'échec.

Il se heurte davantage que la génération précédente au fait que les autorités britanniques lui dictent une politique essentiellement motivée par des considérations de politique britannique, européenne ou impériale qui ne prennent pas en compte les besoins spécifiques du souscontinent $^{21}$. Comme le relève M. McLaren, l'Écosse comme l'Inde britannique appartenaient à ce que l'on appelle parfois le «Second British Empire» et étaient en fin de compte soumises à «the authority of Britain's predominantly English, socially and politically exclusive ruling elite ${ }^{22}$ ». Les élites politiques à Londres avaient coutume de se soucier aussi peu des besoins particuliers de l'une que de l'autre. Plus que jamais depuis le rappel de Wellesley en 1805, le gouvernement britannique veille à affermir son emprise sur le gouvernement de l'Inde, notamment par le biais des nominations politiques aux postes les plus élevés. La politique dictée par Londres vise avant tout à répondre aux attentes de l'opposition

18. Ibid., p. 372.

19. E. Stokes, The English Utilitarians and India, p. 247-248.

20. B. B. Misra, The Administrative History of India, 1834-1947, p. 643.

21. "The British built a territorial raj in India, which had its own imperatives for foreign policy, differing indeed from those of Britain itself. Within India it could contemplate no challenge; on its frontiers it insisted on compliance.» (Nicholas Tarling [éd.], The Cambridge History of Southeast Asia, vol. II : The Nineteenth and Twentieth Centuries, p. 34.)

22. M. McLaren, British India and British Scotland, 1780-1830, p. 6. 
pour éviter que le gouvernement ne soit mis en difficulté au Parlement et à ne pas s'aliéner l'opinion publique.

Colley rappelle les deux facettes de la contribution écossaise : l'une est agressive et sans scrupules, l'autre porte l'héritage des Lumières. Burnes incarne comme ses prédécesseurs cette double identité. Comme eux, il est un carriériste. Il correspond au modèle écossais en ce qu'il utilise ses compétences intellectuelles (comme auteur) et pratiques (comme homme d'action) pour améliorer son statut de citoyen de la Grande-Bretagne. Il sert la Grande-Bretagne et l'Empire, mais à aucun moment il ne perd son identité écossaise qui ne peut qu'être renforcée par les difficultés auxquelles il est confronté. Sa réputation pâtit du désastre que constitue la retraite d'Afghanistan, bien qu'il ait fait savoir de manière officielle comme officieuse qu'il désapprouvait la politique menée. L'évolution très rapide de sa carrière et sa célébrité ont pu susciter des jalousies et son propos a perdu de sa force en raison de certaines des positions qu'il a pu adopter. Lunt avance que l'on a accordé trop d'attention à l'avis de Burnes au début, et pas assez ensuite. Si Burnes recommande de 1833 à la fin de sa carrière de soutenir Dost Mohammad plutôt que Shah Shudja, il évolue au cours de cette période d'une position interventionniste à une position attentiste. Par ailleurs, dès la fin de l'année 1839, Burnes se plaint que ses lettres recommandant de parvenir à un accord avec Dost Mohammad aient été tronquées pour donner l'impression qu'il préconise de mener cette politique avec Shah Shudja ${ }^{23}$. Il dit des propos qui lui sont attribués dans les documents officiels, qui le font apparaître comme étant celui qui a conseillé la politique à laquelle il a en vain tenté de s'opposer, qu'ils sont «pure trickery». Sa disparition prématurée ne lui permet pas de se défendre. En relâchant Dost Mohammad et en le laissant remonter sur le trône de Kaboul en 1843, les autorités britanniques reconnaissent a posteriori la justesse de ses préconisations, quels que soient les reproches qui peuvent lui être faits. Le fait que le gouvernement britannique ait, pour atténuer sa propre responsabilité dans l'échec de la politique afghane, publié une correspondance officielle tronquée (Blue Book de 1839, connu sous le nom de «garbled blue book») est de nature à peser favorablement en faveur de Burnes. Mais vingt ans plus tard, Palmerston, devenu Premier ministre, refusera à son frère James, soutenu par le radical John Bright, le rétablissement de la vérité.

On sait que l'Inde a été un terrain d'expérimentation pour quantité de théories. Burnes est confronté à un décalage accentué par l'éloignement géographique entre les influences qui prévalent au même moment en Inde et en Grande-Bretagne. La gouvernance du sous-continent est

23. J. W. Kaye, Lives of Indian Officers, vol. 2, p. 65. 
soumise à la fois à des influences philosophiques ou morales qui n'opèrent pas ou peu en Grande-Bretagne (le paternalisme notamment) et à d'autres plus spécifiquement britanniques qui, elles, évoluent très vite. Concernant la question afghane, Burnes est l'héritier du paternalisme indien qu'incarnent Malcolm et Elphinstone alors que c'est désormais l'influence de Palmerston qui prévaut à Londres. Wellington lui-même, autorité morale pourtant incontestée sur les questions indiennes ${ }^{24}$, n’a pas gain de cause en 1838 lorsqu'il prévoit que les difficultés politiques seront supérieures aux difficultés militaires.

Jusque dans les années 1830, les valeurs des Lumières écossaises ont, grâce aux administrateurs écossais mais aussi aux philosophes ou théoriciens à Londres, exercé une influence considérable dans le souscontinent. En matière de gouvernance, l'héritage écossais est associé à l'autoritarisme constitutif du paternalisme indien ainsi qu'à l'utilitarisme, au réformisme et au libéralisme des années 1830. Le programme libéral doit beaucoup à Adam Smith, notamment.

En désignant des boucs émissaires pour expliquer l'échec de la politique afghane, le gouvernement britannique comme le Parlement (où s'exercent toutes sortes d'influences, évangélique et mercantile notamment) occultent la responsabilité déterminante qu'ils ont dans le gouvernement de l'Inde. À Londres, l'idée prend forme que le seul bon gouvernement pour l'Inde est le despotisme censément impartial et bienveillant exercé par le gouvernement britannique. Avec le passage de l'autoritarisme, ou despotisme éclairé, indien, au despotisme exercé par Londres, l'influence des valeurs écossaises en matière de gouvernance indienne reçoit un coup d'arrêt et se trouve désormais circonscrite.

\section{Références bibliographiques}

\section{Ouvrages}

BAYLy Christopher Alan, Indian Society and the Making of the British Empire, The New Cambridge History of India, vol. II.1, Cambridge, Cambridge University Press, 1990 (1988).

- Empire and Information, Intelligence gathering and social communication in India, 1780-1870, Cambridge Studies in Indian History and Society, Cambridge, Cambridge University Press, 1996.

24. De 1796 à 1805, Wellington a servi en Inde où son frère, Richard Wellesley, a occupé le poste de gouverneur général de 1798 à 1805. Il n'a ensuite jamais cessé de suivre de près les affaires indiennes. 
Burnes Alexander, Travels into Bokhara... in the years 1831, 32 and 33, Londres, John Murray, 1834, 3 vol.

- Cabool, being a personal narrative of a journey to, and residence in that city, in the years 1836, 7 and 8, Londres, John Murray, 1842.

Colley Linda, Britons: Forging the Nation, 1707-1837, New Haven, Londres, Yale University Press, 1992.

Hopkink Peter, The Great Game: The Struggle for Empire in Central Asia, Londres, John Murray, 1990.

KaYE John William, History of the War in Afghanistan: from the unpublished letters and journals of political and military officers employed in Afghanistan throughout the entire period of British connexion with that country, Londres, William Allen, 1890, 3 vol. [Londres, Richard Bentley, 1851, 2 vol.].

- Lives of Indian Officers: illustrative of the history of the civil and military services of India, Londres, A. Strahan \& Bell and Daldy, 1867, 2 vol.

Lunt James, Bokhara Burnes, New York, Barnes and Noble, 1969.

Marx Karl et Engels Friedrich, Du Colonialisme en Asie: Inde, Perse, Afghanistan, Paris, Mille et Une Nuits, 2002.

MaLaren Martha, British India and British Scotland, 1780-1830, The University of Akron Press, 2001.

Metcalfe Thomas R., Ideologies of the Raj. The New Cambridge History of India, vol. III.4, Cambridge University Press, 1994.

Meyer Karl Ernest et BrysaC Shareen Blair, Tournament Of Shadows: The Great Game and the Race for Empire in Central Asia, Counterpoint, 1999.

Misra Bankey Bihari, The Administrative History of India, 1834-1947, Oxford University Press, 1970.

Stokes Eric, The English Utilitarians and India, Oxford University Press, 1959.

Tarling Nicholas (éd.), The Cambridge History of Southeast Asia, vol. II : The Nineteenth and Twentieth Centuries, Cambridge University Press, 1994 (1992).

YAPP Malcom E., Strategies of British India: Britain, Iran and Afghanistan, 1798-1850, Oxford, Clarendon Press, 1980.

\section{Articles}

STOKes Eric, «Bureaucracy and Ideology: Britain and India in the Nineteenth Century», Transactions of the Royal Historical Society, $5^{\mathrm{e}}$ serie, XXX, 1980, p. 131-156.

Vaughan Géraldine, «Un Empire écossais? L'Écosse et le monde britannique, 1815-1931»[en ligne], Histoire@Politique. Politique, culture, société, no 11, mai-août 2010, disponible sur <www.histoire-politique. $\mathrm{fr} /$ documents / 11/dossier/pdf/HP11_Vaughan_pdf_210510.pdf> [consulté le 8 février 2011]. 